\title{
Ações de Terapia Ocupacional no território da cultura: a experiência de cooperação entre o Museu de Arte Contemporânea da USP (MAC USP) e o Laboratório de Estudos e Pesquisas Arte e Corpo em Terapia Ocupacional*
}

\section{Actions of Occupational Therapy in the territory of culture: a cooperative experience between the Museum of Contemporary Art of USP (MAC USP) and the Laboratory of Studies and Research in Art, Body and Occupation Therapy}

\author{
Sylvio Coutinho ${ }^{1}$, Eliane Dias de Castro ${ }^{2}$, Erika Alvarez Inforsato ${ }^{3}$, \\ Leonardo José da Costa Lima ${ }^{4}$, Ana Tereza Galvanese ${ }^{5}$, \\ Gisele Asanuma ${ }^{5}$, Elizabeth M. F. Araújo Lima²
}

\begin{abstract}
COUTINHO, S.; CASTRO, E. D. de; INFORSATO E. A.; LIMA, L. J. da C.; GALVANESE, A. T.; ASANUMA, G.; LIMA, E. M. F. A. Ações de Terapia Ocupacional no território da cultura: uma experiência de cooperação entre o Museu de Arte Contemporânea da USP (MAC USP) e o Laboratório de Estudos e Pesquisas Arte e Corpo em Terapia Ocupacional. Rev. Ter. Ocup. Univ. São Paulo, v. 20, n. 3, p. 188-192, set./dez. 2009.

RESUMO: Este trabalho se propõe a relatar a experiência de colaboração entre o Laboratório de Estudos e Pesquisas Arte e Corpo em Terapia Ocupacional e o Museu de Arte Contemporânea da USP (MAC USP), que teve início em 1996, focalizando algumas ações significativas e seus desdobramentos para a construção de práticas de Terapia Ocupacional no campo cultural. Durante mais de 10 anos foram desenvolvidas ações conjuntas nas esferas do ensino, da pesquisa e da extensão de serviços à comunidade. A experiência possibilitou o acompanhamento de grupos de idosos e pessoas em situação de vulnerabilidade, em propostas que compreendem o fazer artístico, a apreciação e a contextualização de práticas estéticas na história da arte; propiciou, também, o aprofundamento de estudos sobre arte contemporânea e a compreensão do papel do terapeuta ocupacional na construção de acesso para populações vulneráveis ao campo artístico-cultural.
\end{abstract}

DESCRITORES: Terapia Ocupacional/tendências. Arte. Ação intersetorial. Cultura.

\footnotetext{
* Este artigo é desdobramento do Projeto Fapesp 02/10358-3 e teve aprovação da Comissão de Ética para Análise de Projetos de Pesquisa - CAPPesq, do HC- FMUSP, protocolo de pesquisa 160/03.

1 Arte - educador da Divisão Técnico-científica de Educação e Arte do Museu de Arte Contemporânea da Universidade de São Paulo MAC-USP

${ }^{2}$ Docente do Curso de Terapia Ocupacional do Depto de Fisioterapia, Fonoaudiologia e Terapia Ocupacional da FMUSP.

3 Terapeuta Ocupacional do Curso de Terapia Ocupacional da FMUSP.

${ }^{4}$ Docente do curso de Terapia Ocupacional da Universidade de Sorocaba.

5 Terapeuta ocupacional.

Endereço para correspondência: Curso de Terapia Ocupacional USP. Rua Cipotânea, 51. Cidade Universitária. C.E.P. 05360-160. Butantã. São Paulo. São Paulo. Brasil. E-mail: beth.lima@usp.br
} 


\section{INTRODUÇÃO}

$\mathrm{N}$ a contemporaneidade, experiências e estudos no campo da Saúde e da Cultura apontam para a necessidade da produção de novas tecnologias sócio-culturais e do desenvolvimento de práticas e propostas de políticas públicas culturais que operem sob os princípios da inclusão, equidade e diversidade cultural e proponham soluções criativas e participativas para populações em situação de vulnerabilidade.

A construção dos direitos substanciais - afetivos, relacionais, materiais, habitacionais, produtivos e culturais - vem sendo, progressivamente, compreendida como dimensão essencial da reabilitação e do exercício da cidadania através de práticas de intervenção social que favoreçam o acesso aos meios de formação, criação, difusão e fruição cultural da população local (SARACENO, 1999; BRASIL, 2006).

Segundo a Declaração Universal da Diversidade Cultural, a dimensão cultural é indispensável e estratégica para qualquer projeto de inclusão, considerando que indivíduos e grupos devem ter, garantidas, as condições de criar e difundir suas expressões culturais (BRASIL, 2005).

Nessa perspectiva, o Laboratório de Estudos e Pesquisas Arte e Corpo em Terapia Ocupacional ${ }^{1}$,tem como uma de suas finalidades efetivar parcerias com projetos que desenvolvem ações no campo da arte e da produção cultural, apresentando interfaces com múltiplos aspectos da área da Saúde.

Entre outras iniciativas, o Laboratório mantém, desde 1996, um acordo de Cooperação com a Divisão Técnico-Científica de Educação e Arte do Museu de Arte Contemporânea da Universidade de São Paulo (MAC USP), através do qual são desenvolvidas atividades conjuntas de ensino, pesquisa e extensão universitária, que configuram um importante campo de formação, atuação e reflexão interdisciplinar para as áreas envolvidas. O MAC USP se constitui enquanto campo fecundo de contato com produções artísticas contemporâneas e dispõe de recursos que contribuem para a formação de alunos e profissionais de Terapia Ocupacional em projetos voltados para a inclusão sócio-cultural da população atendida.

O trabalho realizado é avaliado a cada dois anos, quando são atualizadas as ações a serem desenvolvidas no próximo período. Entre as atividades conjuntas desenvolvidas nesses 10 anos, destaca-se a participação de terapeutas ocupacionais no Programa Lazer com Arte para a Terceira Idade, no Programa o Museu e o Público Especial e a proposição conjunta dos Projetos: Acompanhamento de Projetos Artísticos e o Projeto de Monitoria Especial².

Este artigo apresenta, de forma sucinta, a história desta cooperação, o desenvolvimento das ações no Programa Lazer com Arte para a Terceira Idade e do Curso de Especialização Práxis Artística e Terapêutica: interfaces da arte e da saúde, Por fim, são levantadas questões que discutem a construção de práticas de terapia ocupacional no campo cultural.

\section{Como tudo começou}

Em 1994 um grupo de professores do Curso de Terapia Ocupacional da USP procurou o professor Sylvio Coutinho, arte-educador do MAC USP, com interesse em estabelecer uma aproximação com uma instituição do território artísticocultural e com o programa de extensão universitária voltado para a população idosa. A intenção inicial era fortalecer as práticas e os conhecimentos relativos ao trabalho com atividades artísticas que vinham sendo desenvolvidas no campo da Terapia Ocupacional (LIMA, 1996).

Este encontro gerou formas de colaboração de mútuo interesse. Em 1995 foi instaurado um processo de acompanhamento prático dos ateliês do Programa Lazer com Arte para a Terceira Idade (LAPTI), e, em 1996, iniciou-se uma experiência de formação teórico-prática junto a terapeutas ocupacionais, docentes e supervisores de estágio do Curso de Terapia Ocupacional da USP.

A partir dessas primeiras experiências de trabalho conjunto, a formalização do trabalho foi ganhando outras consistências e foi então criado no MAC USP um Curso de Extensão Universitária voltado para a formação de terapeutas ocupacionais no campo das artes plásticas, que viabilizava a utilização de metodologias de ensino de arte com os alunos da graduação de Terapia Ocupacional e com

\footnotetext{
${ }^{1}$ Laboratório do Curso de Terapia Ocupacional do Departamento de Fisioterapia, Fonoaudiologia e Terapia Ocupacional da FMUSP. ${ }^{2}$ O Programa o Museu e o Público Especial foi desenvolvido pela arte-educadora Amanda Tojal e teve a colaboração da terapeuta ocupacional Ana Lúcia Borges da Costa. Os outros dois projetos foram desenvolvidos conjuntamente por profissionais do PACTO e do MAC USP. A Monitoria Especial foi um projeto proposto e desenvolvido conjuntamente por Christiana Moraes e Erika Inforsato; os Ateliês de Acompanhamento de Poéticas Pessoais foram desenvolvidos por Christiana Moraes, Daniela Figueiredo Canguçu, André Nunes e Camila Apostólico.
} 
a população atendida.

Em contrapartida, a participação de terapeutas ocupacionais junto ao LAPTI estruturou-se na atenção à dinâmica grupal, no acompanhamento dos integrantes de forma mais próxima e singularizada e na efetivação de um espaço no qual pudessem ser acolhidas as demandas e questões relativas à saúde e ao processo de envelhecimento. Durante os ateliês, a atuação interdisciplinar, envolvendo profissionais, estagiários e bolsistas dos campos das artes e da saúde, e a observação cuidadosa do envolvimento e da participação dos idosos nas propostas de trabalho artístico, facilitava o processo de criação e restituía aos participantes e ao projeto uma atualização e uma vitalidade necessárias à produção artística (CASTRO, 2001).

Este encontro trouxe para o campo da Terapia Ocupacional referências sobre a história da arte, em especial da arte contemporânea e da abordagem triangular do ensino da arte (BARBOSA, 2008; RIZZI, 2008; PILLAR; VIEIRA, 1992) ${ }^{3}$ o que potencializou novas orientações para a formação e as práticas com as atividades artísticas neste campo e abriu perspectivas de participação sócio-cultural à população atendida.

Esta prática conjunta deu lugar, dois anos depois, ao estabelecimento de um acordo de cooperação institucional entre o MAC USP e o Curso de Terapia Ocupacional da USP, através do Laboratório de Estudos e Pesquisas Arte e Corpo em Terapia Ocupacional da FMUSP, em vigor até hoje, ampliando e fortalecendo composições no âmbito da extensão universitária, abrindo espaço para novas parcerias da terapia ocupacional com outros projetos deste museu.

\section{A colaboração da Terapia Ocupacional junto ao Programa Lazer com Arte para a Terceira Idade}

A proposta do LAPTI é voltada para idosos, aposentados ou não, iniciando-os numa práxis artística contemporânea. As atividades do Programa, centradas em práticas de ateliê, partem da apreciação comentada de obras selecionadas do acervo do MAC USP, em articulação com as informações que contextualizam historicamente obras, artistas e movimentos artísticos da contemporaneidade. Interpretadas as poéticas visuais dos artistas tomados como referência, em suas diversas fases, os procedimentos de ateliê decorrem das possíveis decodificações de suas técnicas, à altura da experimentação dos participantes do Programa. Desencadeia-se, assim, uma relação dialógica sensível entre poéticas do artista e do aprendiz (COUTINHO, 1996).

O LAPTI propicia a atualização cultural do idoso através de visitas a museus, galerias e ateliês de artistas e passeios culturais como espetáculos teatrais, cinemas, palestras e eventos. Partilhados coletivamente, esses eventos redimensionam as experiências culturais e enriquecem as trocas, agregando valor às próprias produções e modos de olhar e circular pelos espaços culturais. A aquisição e ampliação de repertórios no universo da arte e de interlocuções do sujeito com esse universo asseguram a possibilidade de produção cultural dos participantes e do grupo, articuladas às produções atuais, contextualizadas, dialogando com o que está sendo produzido no campo. Anualmente, mostras didáticas dos participantes do LAPTI são organizadas, estimulando trocas e interlocuções com o público em geral.

Com o tempo, estabeleceu-se um certo formato para o trabalho nos ateliês, iniciado com uma dinâmica de grupo coordenada por terapeutas e estudantes de Terapia Ocupacional, seguida das atividades desenvolvidas em ateliê, em que os participantes alternam momentos de apreciação de livros de arte, visitas ao acervo do museu e realização de atividades plásticas. Em geral, ao final das atividades, os alunos expõem o que produziram em um varal e o professor dispõe-se a um processo de avaliação e leitura de cada exercício-obra daquele dia. Em todas essas fases, os participantes são continuamente convidados ao envolvimento com a experiência estética e com a criação, e é nesse eixo que as relações se estabelecem.

Desta forma, o programa se configura como um espaço cultural onde a parceria é tecida a muitas mãos: arteeducador, terapeuta ocupacional, estagiários e assistentes, todos empenhados tanto no processo quanto nas produções nele engendradas, tendo como fio condutor a abordagem triangular de ensino de arte com suas premissas: conhecer, contextualizar e fazer (BARBOSA, 2008; RIZZI, 2008).

\section{A partir da contextualização e de uma apreciação mais acurada, obras e artistas selecionados tornam-se referenciais para a reinterpretação, transformação e interação criativa que vão ajudar na construção das poéticas visuais de cada um dos participantes (COUTINHO, 2007).}

A equipe se defronta constantemente com desafios, entre os quais: funcionar em sinergia; produzir continuamente consensos na ação, e acolher mutuamente as diferentes contribuições dos seus membros.

\footnotetext{
${ }^{3} \mathrm{O}$ termo "metodologia triangular do ensino da arte" foi revisto por Ana Mae Barbosa em seus textos mais recentes, nos quais o substitui por "abordagem triangular" ou "proposta triangular".
} 
Através do trabalho no ateliê, a parceria se aprimora, buscando construir uma experiência transdisciplinar. Trabalhar na perspectiva transdisciplinar significa operar num sistema de referência que tem três bases de sustentação: a abordagem dos problemas pelos diversos níveis de realidade possíveis, a fim de produzir discussões que possam se colocar adiante de concepções unilaterais; a lógica do terceiro termo incluso, isto é, a conciliação possível de termos contraditórios, para escapar à concepção simplista de verdadeiro ou falso; e o reconhecimento da complexidade dos problemas abordados (GALHEIGO, 1999, p. 50)

\section{Os Cursos de Extensão e de Especialização promovidos conjuntamente}

O Curso de Extensão Universitária "Práxis Artística para terapeutas ocupacionais", foi desenvolvido durante quatro semestres, com encontros semanais às sextas-feiras à tarde, com a participação dos professores e supervisores de estágio ligados ao Curso de Terapia Ocupacional da USP e aberto a outros terapeutas ocupacionais interessados em formação no campo das artes.

Num primeiro momento foi proposta uma imersão no campo das artes, conhecendo artistas, movimentos, procedimentos e mergulhando no fazer artístico, no contato com as cores, as tintas, sua plasticidade, seu encantamento. Aos poucos foram sendo trazidas para os encontros questões relativas às relações entre a prática vivenciada, a promoção da saúde e o desenvolvimento de práticas terapêuticas relacionadas às atividades artísticas.

Com o aprofundamento dessas questões e o interesse em pesquisar e promover uma formação interdisciplinar na interface da arte e da saúde esta experiência ganhou contornos acadêmicos mais ampliados, desembocando na promoção do Curso de Especialização "Práxis Artística e Terapêutica: interfaces da arte e da saúde". Este curso constituiu-se em uma experiência de formação em nível de pós-graduação lato-senso voltada para terapeutas ocupacionais e outros profissionais dos campos das artes e da saúde. Inicialmente com duração de um ano, na segunda turma foi ampliado para dois anos tendo em vista a complexidade e a diversidade dos conhecimentos envolvidos e a necessidade de um tempo formativo maior, para a elaboração e aprofundamento desses conhecimentos.

O Curso foi organizado em dois eixos principais: um eixo prático de atividades artísticas, compreendendo práticas em ateliê de artes plásticas e artes do movimento, e um eixo histórico-conceitual sobre a constituição e o desenvolvimento de práticas na interface entre arte e promoção da saúde. Na dinâmica de todas as aulas buscava- se integrar e articular os conteúdos trabalhados nos dois eixos. Referenciais dos campos das artes, da arte-educação, da terapia ocupacional e da reabilitação psicossocial, compunham a perspectiva teórica interdisciplinar desta formação, envolvendo discussões acerca da história da arte, dos instrumentais teóricos para a prática terapêutica com atividades artísticas e das práticas contemporâneas na fronteira entre os campos. Além disso, o curso previa também o trabalho de orientação de projetos de pesquisa e seminário temáticos.

A experiência foi desenvolvida entre os anos de 1998 a 2002, tendo formado três turmas, e revelou um fecundo campo interdisciplinar de práticas e saberes, apontando novas direções para a as ações de inclusão sócio-cultural e para a pesquisa em Terapia Ocupacional, de modo a subsidiar o desenvolvimento de componentes curriculares em programa de pós-graduação senso-estrito na área.

\section{CONCLUSÃO}

O campo de atuação na interface da cultura com a saúde é um campo em ampliação e desenvolvimento na Terapia Ocupacional. Cada vez mais, terapeutas ocupacionais têm contribuído para as práticas e programas desenvolvidos em equipamentos e projetos culturais de modo a promover uma rede horizontal de articulação, recepção e disseminação de informações e garantir o acesso de populações em situação de vulnerabilidade aos recursos e programas de formação, difusão e fruição cultural.

Neste âmbito, o conjunto de ações desenvolvidas em terapia ocupacional pressupõe o enfrentamento de questões sócio-políticas do país que são vividas nas práticas de inclusão sócio-cultural. Estas ações propiciam a produção de saberes e geram modelos de ação inovadores, fortalecem iniciativas no campo da cultura e sinalizam a construção de uma política cultural democrática, voltada para a ampliação dos direitos das pessoas e para o exercício da cidadania.

Esse acúmulo de experiências práticas cria também um avanço nas discussões acadêmicas, sistematizações e trocas de experiências, no intuito de conduzir à construção de modelos teóricos e metodológicos que subsidiem o desenvolvimento de pesquisas em Terapia Ocupacional na interface arte e saúde e favoreçam a criação e implantação de políticas públicas que incluam o terapeuta ocupacional nas diferentes formas de produção cultural. Considera-se que questões relacionadas ao campo da saúde e da cultura devem atravessar-se mutuamente, produzindo um enfrentamento da exclusão social.

A experiência de cooperação entre o Laboratório de Estudos e Pesquisa Arte e Corpo em Terapia Ocupacional 
e o MAC USP tem se constituído em uma importante referência para o desenvolvimento deste campo de interface, propiciando a estudantes e profissionais das artes e da terapia ocupacional espaço para a experimentação e invenção de estratégias de atuação. A composição desta parceria potencializa as atividades desenvolvidas pelo Laboratório no que concerne às inovações no ensino de Graduação, sensibilizando e instrumentalizando os alunos para as ações no campo artístico-cultural, e fortalece iniciativas de extensão de serviços à comunidade nesta Universidade.
Propicia, ainda, o desenvolvimento de pesquisas que visam a atualização e reflexão sobre os recursos das linguagens artísticas na perspectiva da Terapia Ocupacional.

A participação de terapeutas ocupacionais e estudantes de terapia ocupacional em programas de um museu de arte possibilita, ainda, a compreensão do papel do terapeuta ocupacional na construção de acesso para populações vulneráveis, ampliando a atenção em saúde para além dos modelos institucionais tradicionais em direção ao campo social, artístico e cultural.

COUTINHO, S.; CASTRO, E. D. de; INFORSATO E. A.; LIMA, L. J. da C.; GALVANESE, A. T.; ASANUMA, G.; LIMA, E. M. F. A. Actions of Occupational Therapy in the territory of culture: a cooperative experience between the Museum of Contemporary Art of USP (MAC USP) and the Laboratory of Studies and Research in Art, Body and Occupation Therapy. Rev. Ter. Ocup. Univ. São Paulo, v. 20, n. 3, p. 188-192, set./dez. 2009.

\begin{abstract}
This article's aim is to report a collaborative experience, started in 1996, between the Laboratory of Studies and Research in Art, Body and Occupation Therapy and the Museum of Contemporary Art of USP (MAC USP), by focusing on some actions and their unfolding for the construction of Occupational Therapy practices in the cultural field. For 10 years, joint actions in teaching, researching and extending services to the community were developed. The experience made it possible to follow up groups of elderly people and people in vulnerability, in proposals that articulate doing art, appreciate and contextualize artistic practices in art history. It made possible, also, deepen studies on contemporary art and the comprehension of occupational therapists' role to build up the access of vulnerable populations to the artistic-cultural field.
\end{abstract}

KEY WORDS: Occupational therapy/trends. Art. Intersectorial action. Culture.

\title{
REFERÊNCIAS
}

BARBOSA, A. M. (org). Ensino da arte: memória e história. São Paulo: Perspectiva, 2008.

CASTRO, E. D. Atividades artísticas e terapia ocupacional: construção de linguagens e inclusão social. 2001.Tese (Doutorado). Escola de Comunicações e Artes da Universidade de São Paulo. São Paulo, 2001.

COUTINHO, S. Lazer com arte para a terceira idade. Disponível em: <http://www.macvirtual.usp.br. Acesso em: 31 jan. 2007.

COUTINHO, S. Programa lazer com arte para a terceira idade. Projeto do Fundo de Cultura e Extensão Universitária - PróReitoria de Cultura e Extensão Universitária da USP, 1996.

GALHEIGO, S. M. A Transdisciplinaridade enquanto princípio e realidade das ações de saúde. Rev. Ter. Ocup. Univ. São Paulo, v. 10, n. 2/3, p.49-54, 1999.

LIMA, E. M. F. A. Terapia Ocupacional: um território de fronteira?
Rev. Ter. Ocup. Univ. São Paulo, v. 8, n. 2-3, p. 98-101, 1997.

BRASIL. Ministério da Cultura. Plano Nacional de Cultura. Brasília: Ministério da Cultura, 2005.

BRASIL. Ministério da Cultura. Programa Cultura Viva. Brasília: Ministério da Cultura, 2006. Disponível em: $<$ http://www.cultura. gov.br/vultura_viva>.

PILLAR, A.; VIEIRA, D.. O vídeo e a metodologia triangular no ensino da arte. Porto Alegre: UFRS/IOCHPE, 1992

RIZZI, M. C. S. L. Reflexões sobre a abordagem triangular no ensino da arte. In: BARBOSA, A. M. (org). Ensino da arte: memória e história. São Paulo: Perspectiva, 2008.

SARACENO, B. Libertando Identidades: da reabilitação psicossocial à cidadania possível. Belo Horizonte: Te Corá Editora/ Instituto Franco Basaglia, 1999. 